\section{Sarcoidosis Presenting as Multifocal Bursitis}

Sir,

Sarcoidosis is a systemic inflammatory disease that can occur anywhere in the body. ${ }^{1}$ Clinical manifestations of sarcoidosis vary according to the site of involved inflammatory lesion. The most common organs of involvement are lung and skin. Musculoskeletal sarcoidosis rarely occurs; however, several cases have been reported.2,3 It is not easy to diagnose sarcoidosis if only the musculoskeletal system shows features of sarcoidosis involvement. Here, we report a case of sarcoidosis with myositis that started with multiple bursitis.

A 37-year man presented with swelling of both elbows, wrists, knees, ankle joints and hip; and pain that started two months ago. Physical examination showed soft movable masses with some tenderness at the bilateral elbows, wrists, and hip. The chest and involved joint $X$-rays showed no abnormal findings. The musculoskeletal ultrasound showed bursa enlargement with synovial villous formation in each involved joint. The laboratory evaluation revealed a white blood cell count of 9,900 cells $/ \mathrm{mm}^{3}$ with an elevated eosinophil percentage of $17.6 \%$, erythrocyte sedimentation rate (15 $\mathrm{mm}$ after first hour), C-reactive protein $(20.9 \mathrm{mg} / \mathrm{L})$, and angiotensin converting enzyme (ACE) level (>150 U/L). The rheumatoid factor and the anti-citrullinated protein antibody levels were within normal range. Musculoskeletal ultrasonography revealed a fusiform mass with an echogenic centre surrounded by a hypoechoic rim below the piriformis; and a grade-2 signal was observed on power Doppler (Figure 1). The biopsy finding of the synovial membrane of the ankle revealed nonspecific lymphocytic infiltration with papillary hyperplasia.

Although the histopathologic examination showed no evidence of granuloma, clinically; sarcoidosis was suspected and prednisolone treatment was started. Thereafter, symptoms and laboratory tests deteriorated or improved according to the steroid dose. Hip MRI showed diffusely increased T2 signal intensity with enhancement in the entire bilateral hip and proximal thigh muscles, mainly in the peripheral portion and intermuscular fascia (Figure 2). Histopathologic examination of proximal thigh lesion showed chronic inflammation with heavy histiocytic and moderate lymphoplastic infiltration and granuloma; findings were consistent with sarcoidosis.

In this patient, a granuloma was not found in the bursa biopsy, but clinical and radiological findings were suspicious. There were unexplained multiple bursitis,

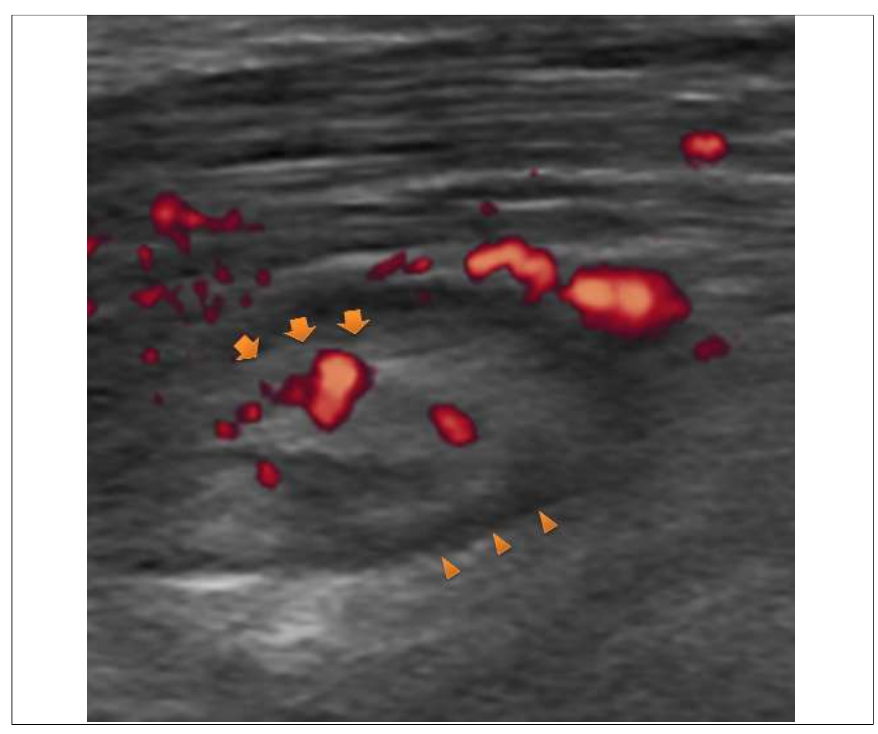

Figure 1: Musculoskeletal ultrasonography. Anterior longitudinal view of the thigh showing active inflammatory tissue. A fusiform, well-defined soft tissue mass (arrows) with an echogenic center surrounded by a hypoechoic rim (arrow-heads) below the priformis.

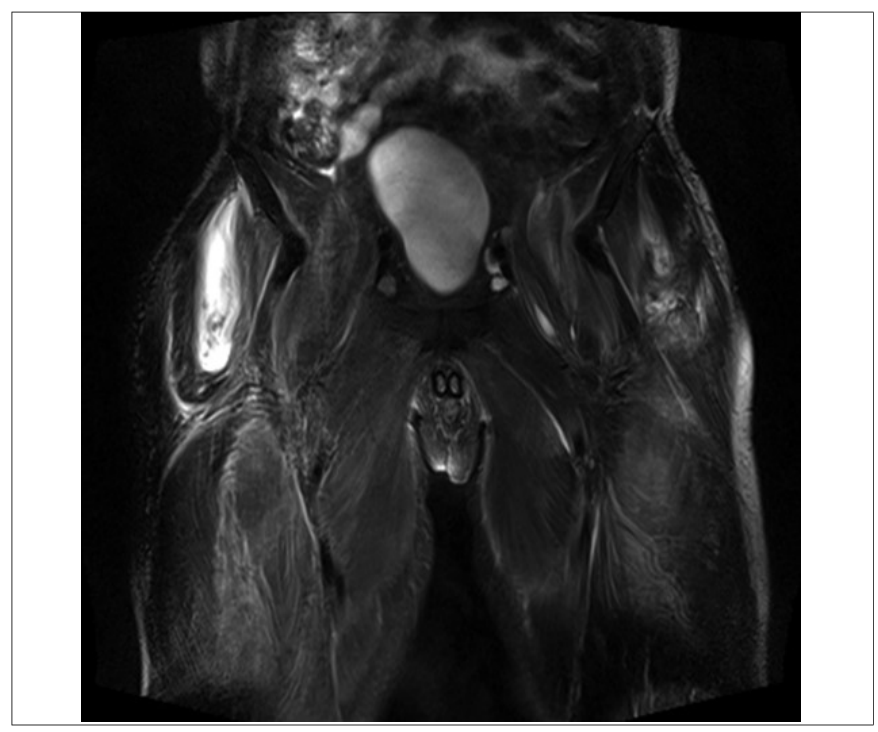

Figure 2: Coronal hip MRI showing diffuse increased T2 signal intensity with enhancement in the entire bilateral hip and proximal thigh muscles, mainly in the peripheral portion and intermuscular fascia. The most severe involvement is in the right piriformis muscle and intermuscular fat plane of the bilateral gluteus muscles.

elevated ACE levels, and a response to steroids. Eventually, it invaded the muscle tissue and showed characteristic imaging and histologic findings. This case illustrates that subcutaneous sarcoidosis may involve multiple bursa in the absence of sarcoidosis in the thorax and skin. When the cause of bursitis is unclear, the possibility of a sarcoidosis should be considered. It is necessary to ascertain other indications by examining whether other organs have sarcoidosis and/or by checking the ACE levels; and if possible, a biopsy should be performed. 


\section{REFERENCES}

1. Yee AM. Sarcoidosis: Rheumatology perspective. Best Prac Res Clin Rheumatol 2016; 30:334-56.

2. Ruangchaijatuporn T, Chang EY, Chung CB. Solitary subcutaneous sarcoidosis with massive chronic prepatellar bursal involvement. Skeletal Radiol 2016; 45:1741-5.

3. Orandi AB, Eutsler E, Ferguson C, White AJ, Kitcharoensakkul M. Sarcoidosis presenting as granulomatous myositis in a 16year-old adolescent. Pediatr Rheumatol Online J 2016; 14:59.
Jae Hyun Jung, Gwan Gyu Song and Jae-Hoon Kim

Department of Internal Medicine, Division of Rheumatology, Korea University Guro Hospital, Seoul, Korea

Correspondence: Dr. Jae-Hoon Kim, Department of Internal Medicine, Division of Rheumatology, Korea University Guro Hospital, 148, Gurodong-ro, Guro-gu, Seoul, 08308, Korea E-mail:visionkjh@naver.com

Received: May 31, 2018; Accepted: November 02, 2018 …….... 